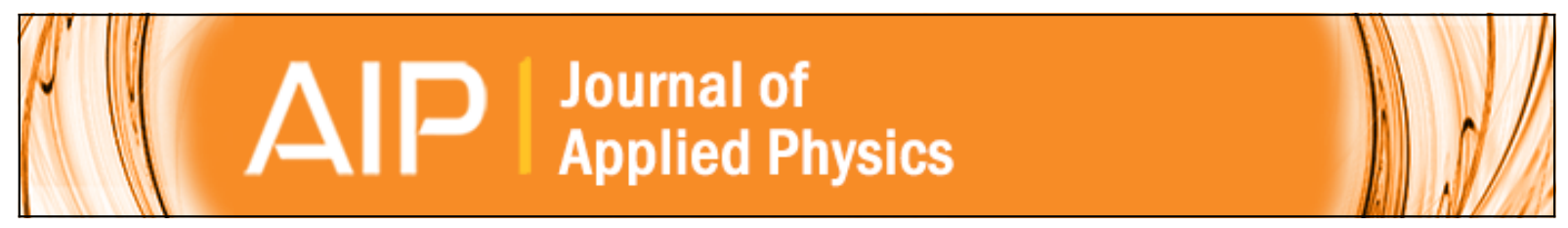

\title{
Magnetoelectric properties of core-shell particulate nanocomposites
}

Rashed A. Islam, Vishwas Bedekar, Narayan Poudyal, J. Ping Liu, and Shashank Priya

Citation: Journal of Applied Physics 104, 104111 (2008); doi: 10.1063/1.3013437

View online: http://dx.doi.org/10.1063/1.3013437

View Table of Contents: http://scitation.aip.org/content/aip/journal/jap/104/10?ver=pdfcov

Published by the AIP Publishing

\section{Articles you may be interested in}

Design tunable materials: Ferroelectric-antiferroelectric composite with core-shell structure

Appl. Phys. Lett. 105, 252901 (2014); 10.1063/1.4905036

Controlled self-assembly of multiferroic core-shell nanoparticles exhibiting strong magneto-electric effects Appl. Phys. Lett. 104, 052901 (2014); 10.1063/1.4863690

Multiferroic and magnetoelectric properties of core-shell CoFe 204 @ BaTiO 3 nanocomposites Appl. Phys. Lett. 97, 062904 (2010); 10.1063/1.3478231

Effect of composition on coupled electric, magnetic, and dielectric properties of two phase particulate magnetoelectric composite

J. Appl. Phys. 101, 014109 (2007); 10.1063/1.2404773

Enhanced magnetoelectric effect in core-shell particulate composites

J. Appl. Phys. 99, 08J503 (2006); 10.1063/1.2165147

MIT LINCOLN

LABORATORY

CAREERS

Discover the satisfaction of innovation and service

to the nation
- Space Control

- Air \& Missile Defense

- Communications Systems \& Cyber Security

- Intelligence, Surveillance and

Reconnaissance Systems

\section{LINCOLN LABORATORY}

MASSACHUSETTS INSTITUTE OF TECHNOLOGY

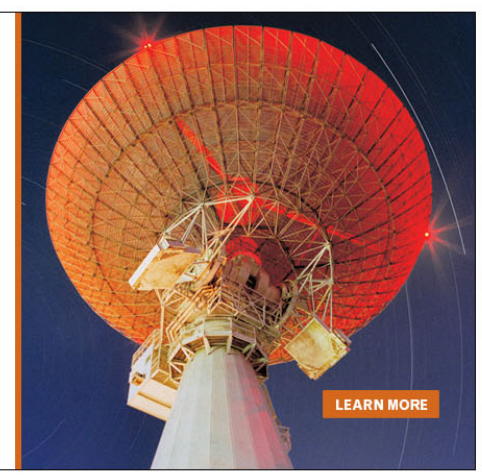




\title{
Magnetoelectric properties of core-shell particulate nanocomposites
}

\author{
Rashed A. Islam, ${ }^{1}$ Vishwas Bedekar, ${ }^{1}$ Narayan Poudyal, ${ }^{2}$ J. Ping Liu, ${ }^{2}$ and \\ Shashank Priya ${ }^{3, a)}$ \\ ${ }_{1}^{1}$ Department of Materials Science and Engineering, UT Arlington, Texas 76019, USA \\ ${ }^{2}$ Department of Physics, UT Arlington, Texas 76019, USA \\ ${ }^{3}$ Materials Science and Engineering, Virginia Tech, Blacksburg, Virginia 24061, USA
}

(Received 28 June 2008; accepted 20 September 2008; published online 20 November 2008)

\begin{abstract}
In this study, we report results on magnetoelectric (ME) core-shell $\mathrm{Pb}(\mathrm{Zr}, \mathrm{Ti}) \mathrm{O}_{3}(\mathrm{PZT})-\mathrm{NiFe}_{2} \mathrm{O}_{4}$ (NF) particulate nanocomposites. NF particles forming the shell had size in range of $20-30 \mathrm{~nm}$. The grain size of sintered nanocomposites was found to be in the range of 500-800 $\mathrm{nm}$. The sintered nanocomposite exhibited piezoelectric coefficient $\left(d_{33}\right)$ of $60 \mathrm{pC} / \mathrm{N}$, dielectric constant of 865 , and ME coefficient of $195 \mathrm{mV} / \mathrm{cm}$ Oe. High ME coefficient was observed for wide range of dc bias magnetic field. This approach of fabricating layered composite has a promise to provide large ME coefficients in particulate sintered structures. (C) 2008 American Institute of Physics.
\end{abstract}

[DOI: 10.1063/1.3013437]

\section{INTRODUCTION}

Magnetoelectric (ME) coupling in piezoelectricmagnetostrictive composites is dependent upon the efficiency of strain transfer across the interface and requires a strong interphase elastic interaction. ${ }^{1-3}$ Particulate (3-0) and layered $(2-1,2-2) \mathrm{ME}$ composites have been reported in literature, which provide varying range of coupling by tailoring the magnitude of strain. ${ }^{4-8}$ Results of Dong et al. ${ }^{9}$ have shown that a 2-1 composite structure with piezoelectric fibers embedded between the high permeability Metglas sheets provides giant magnitude of ME coefficient. However, laminate composites involve multiple step assembly process and are sensitive to the changes in configuration. Thus, our research emphasis has been on enhancing the ME coefficient in bulk sintered composites. The challenges associated with sintered composites are (i) reducing the diffusion across interface, (ii) reducing elastic softening at the interface, and (iii) reducing connectivity of the magnetostrictive phase without compromising the contact area with piezoelectric phase.

All these challenges can be addressed by synthesizing sintered composites from core-shell particles where piezoelectric phase is the core and magnetostrictive phase is the shell. ${ }^{10,11}$ The elastic coupling can be maximized by having coherent response from the magnetostrictive phase under $\mathrm{dc}$ bias such that the stress on the piezoelectric lattice across the grains is in phase with each other. This is only possible if there is uniform distribution of the magnetostrictive phase with respect to the piezoelectric matrix. This core-shell composite has the possibility to provide effective interphase exchange coupling originating from the maximized interphase contact. The aim of this work is to synthesize a core-shell particulate composite of $\mathrm{Pb}(\mathrm{Zr}, \mathrm{Ti}) \mathrm{O}_{3}(\mathrm{PZT})$ and $\mathrm{NiFe}_{2} \mathrm{O}_{4}$ $(\mathrm{NF})$, and characterize its ME property.

\section{EXPERIMENTAL}

PZT particles corresponding to formulation $\mathrm{Pb}\left(\mathrm{Zr}_{0.52} \mathrm{Ti}_{0.48}\right) \mathrm{O}_{3}$ were synthesized using conventional ce-

${ }^{a)}$ Electronic mail: spriya@vt.edu. ramic processing route. Nano-NF particles were synthesized by chemical synthesis technique using the following processing steps. A mixture of $256 \mathrm{mg}$ of $\mathrm{Ni}(\mathrm{acac})_{2}$ and $500 \mathrm{mg}$ of 1,2-hexadecanediol was added to a $125 \mathrm{ml}$ European flask containing a magnetic stir bar. Benzyl ether $20 \mathrm{ml}$ was then transferred into the flask and the contents were stirred while purging with $\mathrm{N}_{2}$ for $20 \mathrm{~min}$ at room temperature. The flask was then heated to $120{ }^{\circ} \mathrm{C}$ and the temperature was held for $20 \mathrm{~min}$. During this time, $0.2 \mathrm{mmol}$ of $\mathrm{Fe}(\mathrm{CO})_{5}$ was injected into the flask while the $\mathrm{N}_{2}$ purging continued. After $1 \mathrm{~min}, 1$ $\mathrm{ml}$ of oleic acid and $1 \mathrm{ml}$ of oleylamine were injected and the mixture was maintained under $\mathrm{N}_{2}$ blanket and heated to $160{ }^{\circ} \mathrm{C}$ at a rate of $5^{\circ} \mathrm{C}$ per minute where it was held for 10 min. The flask was maintained at the refluxing temperature of $295{ }^{\circ} \mathrm{C}$ for $30 \mathrm{~min}$ before cooling down to room temperature under the $\mathrm{N}_{2}$ blanket. The size of $\mathrm{NiFe}_{2} \mathrm{O}_{4}$ was controlled by varying the amount of surfactants and heating rates. When faster heating rate and more surfactants was used bigger NF particles were obtained.

Coating of $\mathrm{NiFe}_{2} \mathrm{O}_{4}$ nanoparticles on PZT was done using similar recipe as that for synthesizing the NF nanoparticles. PZT particles were added in the beginning of synthesis keeping the other parameters constant. Amount of PZT particles was varied from 0.5 to $2 \mathrm{~g}$ in order to vary the coating thickness and obtain different ratio of NF to PZT. Three different ratios of NF nanoparticles to PZT were used corresponding to: $0.5 \mathrm{~g}(\mathrm{NF})$ to $2 \mathrm{~g}$ (PZT), $0.5 \mathrm{~g}$ (NF) to $1.5 \mathrm{~g}$ (PZT), and $0.5 \mathrm{~g}(\mathrm{NF})$ to $1 \mathrm{~g}(\mathrm{PZT})$. According to Transmission Electron Microscope (TEM) observation shown later in the manuscript the ratio of $0.5 \mathrm{~g}(\mathrm{NF})$ to $2 \mathrm{~g}$ (PZT) provides uniform and consistent core-shell structure. Thus, all the samples used in this study correspond to the composition of PZT-20 wt \% NF. The core-shell powders $(0.25 \mathrm{~g})$ were pressed with a top and bottom layer of PZT $(0.1 \mathrm{~g})$ in a $\frac{1}{4}$ in. die under hydraulic pressure of $2 \mathrm{kpsi}$ followed by cold isostatic pressure of 30 psi. The compact was sintered at low temperature of $950{ }^{\circ} \mathrm{C}$ and long holding time of $5 \mathrm{~h}$. The thickness of the top and bottom layer PZT in the sintered and polished composites used for measurement was $100 \mu \mathrm{m}$. 

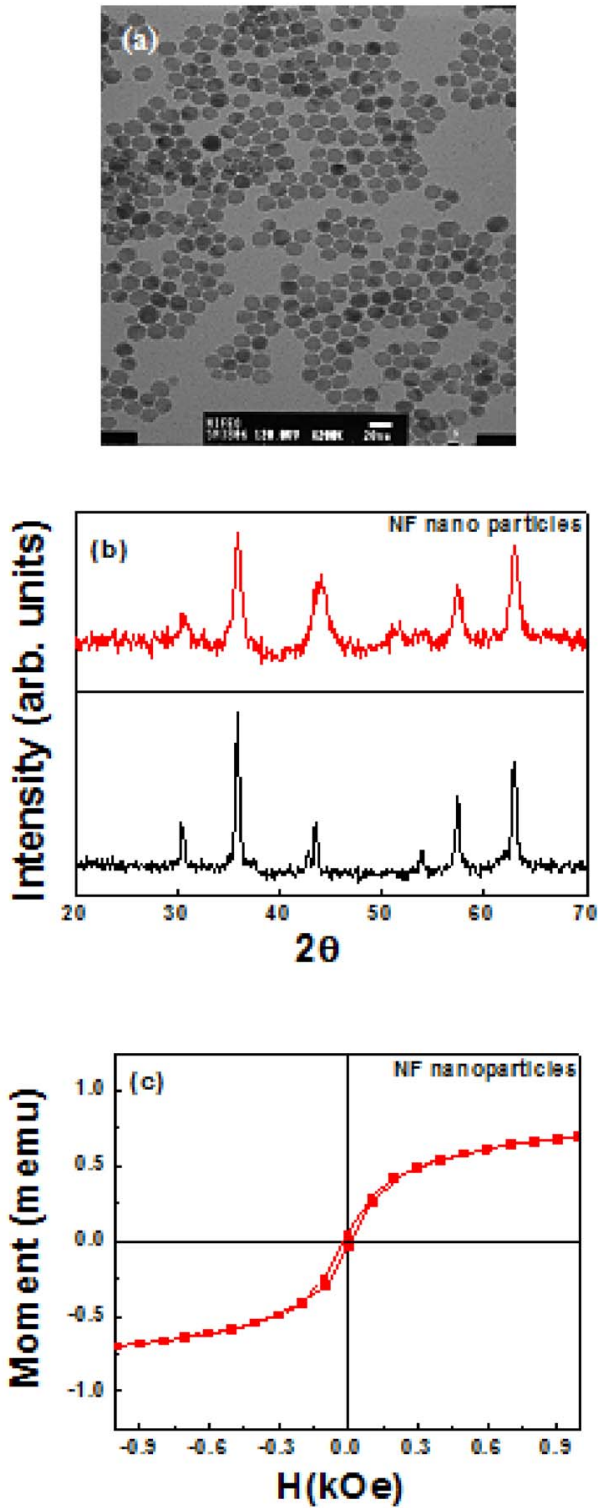

FIG. 1. (Color online) (a) TEM image, (b) x-ray diffraction pattern, and (c) magnetic properties of NF nanoparticles.

In order to perform piezoelectric measurements, an $\mathrm{Ag} / \mathrm{Pd}$ electrode was applied on the samples. The specimens were poled under a dc field of $2.5 \mathrm{kV} / \mathrm{mm}$ for $20 \mathrm{~min}$ in a silicone oil bath at $80-120^{\circ} \mathrm{C}$. During poling there was no rise in current indicating high resistivity of the sintered samples. The piezoelectric constant was measured by APC YE 2730A $d_{33}$ meter. The magnetic properties of the sintered samples were measured at room temperature by using alternating gradient force magnetometer. ME coefficient $(d E / d H)$ was measured by applying an ac magnetic field at $1 \mathrm{kHz}$ and 1 Oe amplitude $(H)$ under varying dc magnetic bias. The ac magnetic field was generated by a Helmholtz coil powered by Agilent 3320 function generator. The output voltage generated from the composite was measured using a SRS DSP lock-in amplifier (model SR 830).

\section{RESULTS AND DISCUSSION}

Figure 1(a) shows the TEM image of nanosize NF par- ticles. The size of the particles was found to be in the range of 20-30 nm with hexagonal shape. Figure 1(b) shows the $\mathrm{x}$-ray diffraction pattern of NF nanoparticles (red) compared with that of large size particles (black), both exhibiting spinel structure. The peak broadening occurred as the particle size became smaller. Figure 1(c) shows the magnetic hysteresis loop of NF nanoparticles. Coercive field and magnetization was found to be lower in comparison with the large size particles, as we will discuss later. From Figs. 1(b) and 1(c), it can be seen that excellent structural and magnetic properties in NF nanoparticles can be achieved using the chemical synthesis method.

Figure 2(a) shows the SEM images of PZT-NF core shell structure. Energy Dispersive Spectroscopy (EDS) spot analysis on these particles showed presence of both PZT and NF. Figure 2(b) shows the TEM image of PZT particle coated with NF nanoparticles. The difference in contrast was clearly observed for two different sizes of particles. TEM investigations showed that the NF nanoparticle shell over the PZT core was 2-3 layers in thickness. Figure 2(c) shows the low magnification SEM image of PZT-NF core-shell structure. The microstructure of the sintered composite was dense with very small fraction of porosity. The grain size of the composite was found to be in the range of 500-800 $\mathrm{nm}$. Inset of Fig. 2(c) shows a magnified view of core-shell grains. TEM analysis revealed that the shell was present at the grain boundary of large fraction of grains. The coating thickness was equivalent to 2-3 layers, which varies from $40-70 \mathrm{~nm}$. The size of nanoparticles in the shell was larger than the as-synthesized nanoparticles due to high temperature sintering process.

The grain size of PZT plays an important role on the magnitude of $\mathrm{ME}$ coupling. Recent results in the composite system of $0.8 \mathrm{~Pb}\left(\mathrm{Zr}_{0.52} \mathrm{Ti}_{0.48}\right) \mathrm{O}_{3}-0.2 \mathrm{Ni}_{0.8} \mathrm{Zn}_{0.2} \mathrm{Fe}_{2} \mathrm{O}_{4}$ have shown that as the piezoelectric grain size increases the overall resistivity, piezoelectric, dielectric, and ferroelectric property of the composite increases and saturates above 600 $\mathrm{nm} .{ }^{12}$ Below $200 \mathrm{~nm}$ average grain size, piezoelectric and dielectric properties decrease rapidly. This can be explained by taking into account the effect of surface bond contraction and domain wall mobility. SEM analysis combined with the surface tension measurement indicate that the surface bond contraction due to small size induces a compressive stress on the inner part of a grain and this effect plays an important role in ferroelectric materials in the nanometer size range. The induced stress causes decrease in Curie temperature and spontaneous polarization with decreasing grain size. The domain wall contribution has an opposite effect as compared with the surface bond contraction induced effect. When the grain size decreases to a value comparable to the width of domain walls, pinning points develop inside the grains and the domain wall motion is inhibited. The reduced wall mobility causes a decrease in the relative permittivity. The measured value is a competition between the increase in relative permittivity by the surface bond contraction effect and its decrease by the domain wall pinning effect. Further it has been shown that $\mathrm{ME}$ coefficient of the $0.8 \mathrm{~Pb}\left(\mathrm{Zr}_{0.52} \mathrm{Ti}_{0.48}\right) \mathrm{O}_{3}-0.2 \mathrm{Ni}_{0.8} \mathrm{Zn}_{0.2} \mathrm{Fe}_{2} \mathrm{O}_{4}$ composite exhibited a rapid change below grain size of $200 \mathrm{~nm}$ and 

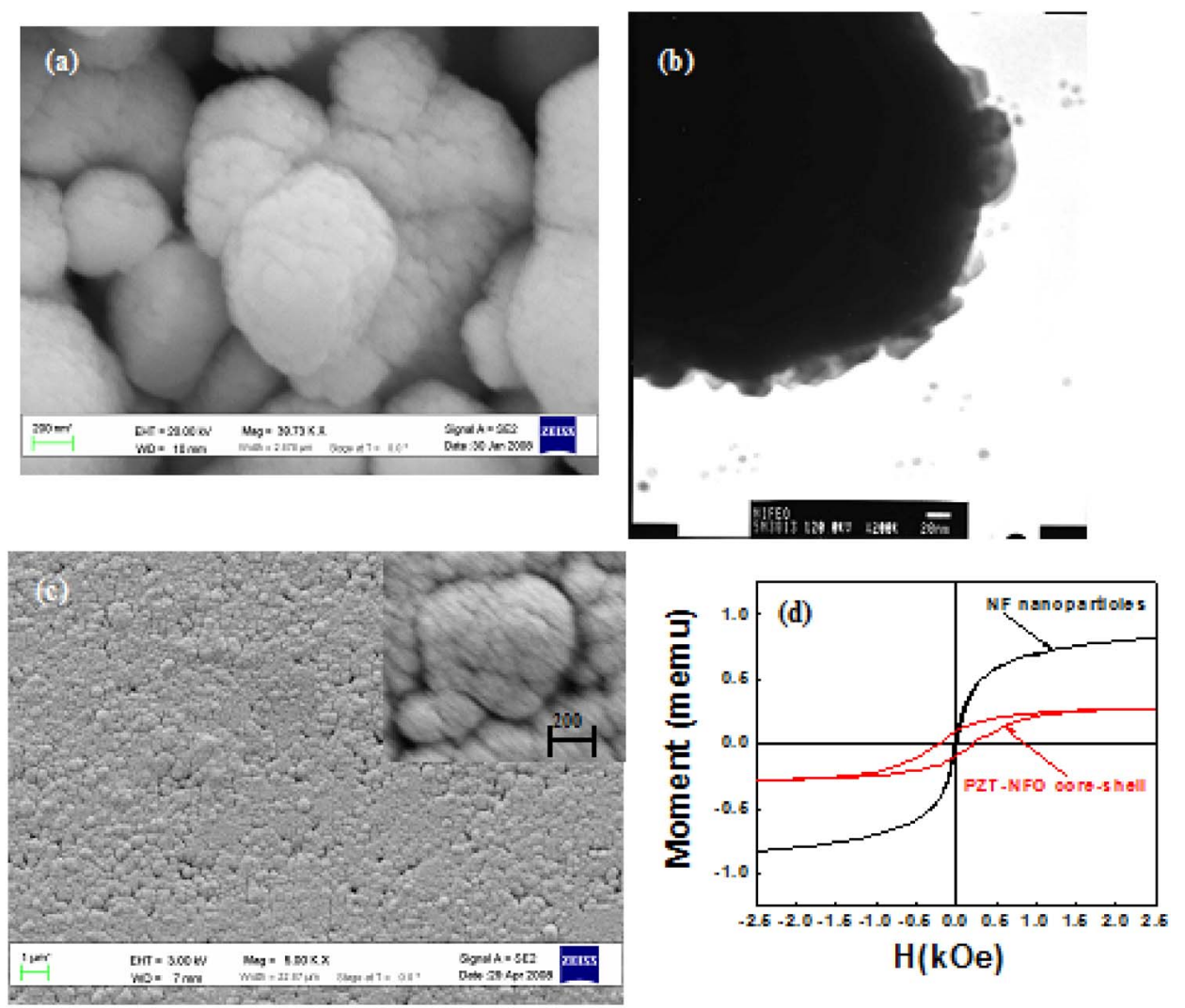

FIG. 2. (Color online) Micrographs for PZT-NF core-shell structure, (a) SEM and (b) TEM, (c) SEM microstructure of sintered PZT-NF core shell structure, and (d) comparison of magnetic hysteresis property of NF nanoparticles and PZT-NF core-shell structure. was found to saturate above $600 \mathrm{~nm}$ to a value of $155 \mathrm{mV} / \mathrm{cm}$ Oe. ${ }^{12}$ Based on these results, the grain size of piezoelectric phase in this study was in the optimum range. The NF layer thickness was optimized to maintain the coreshell structure in sintered composite. Further, the smaller shell thickness will provide higher resistivity in the sintered composite and reduce the additional misfit strain caused by different striction of the adjacent phases. At 2-3 layer thickness of the NF particles with size ratio $\left(r_{\mathrm{NF}}: r_{\mathrm{PZT}}\right)$ in the range of $0.1-0.05$, a well sintered core-shell structure was obtained with high interface bonding.

Figure 2(d) shows the magnetic properties of as synthesized NF nanoparticles and PZT-NF core-shell structure. One major difference can be immediately noted in this figure related to increase in coercive field as compared to the nanoparticles. For PZT-NF core-shell particles the coercive field was found to be 216 Oe whereas for as synthesized nanoparticles this magnitude was significantly lower. This may be attributed to the increase in size of NFO shell during sintering process. As particle size decreases, coercivity decreases and for very low value of particle size ferromagnetic to superparamagnetic transition occurs. ${ }^{13,14}$ It has been shown in literature that with increasing particle size from $1 \mathrm{~nm}$ and above, the magnitude of coercive field increases and reaches maxima before dropping again with further increase in size. The particle size where maximum in coercive field occurs corresponds to the size of single domain particle. The size of shell in the sintered composite may be close to the single domain particle size and hence the coercive field observed was high.

Figure 3(a) shows the TEM image of grain structure for sintered PZT-NF composite and Fig. 3(b) shows the variation
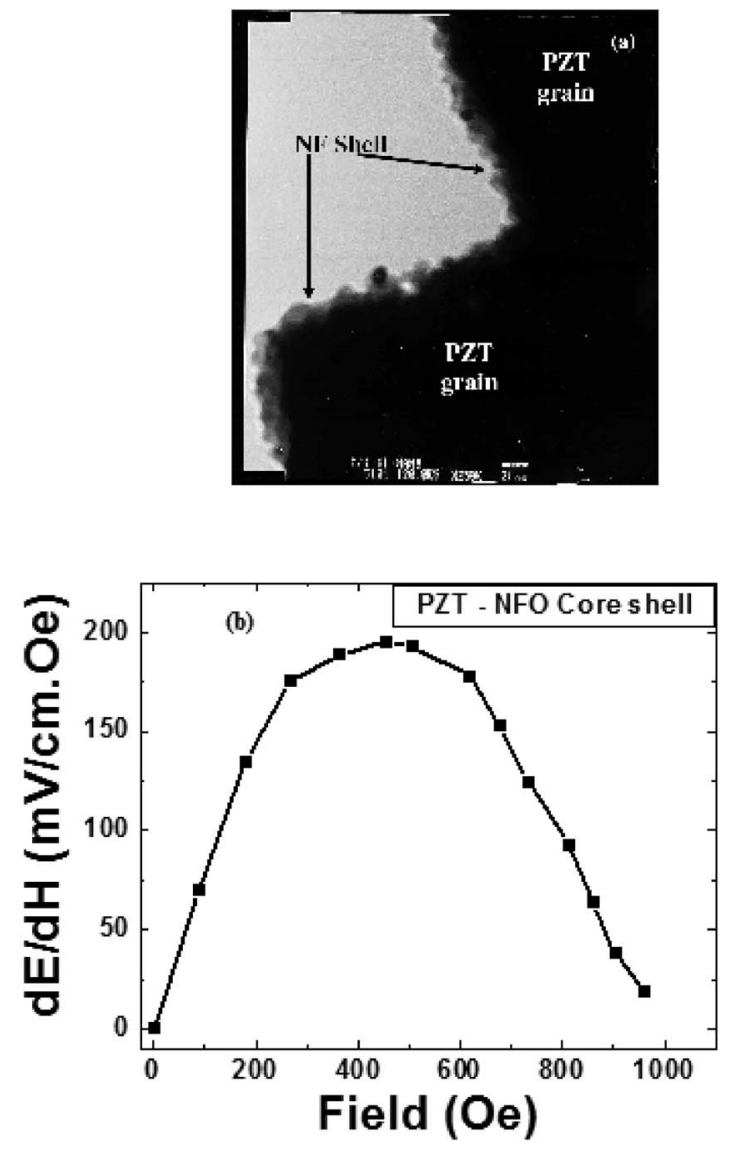

FIG. 3. (a) TEM image of sintered core-shell structure (b) ME coefficient as a function of dc bias field for sintered PZT-NF core shell structure. 
in $\mathrm{ME}$ coefficient as a function of dc bias. The piezoelectric $\left(d_{33}\right)$ and dielectric properties were measured on aged samples. The composite had $d_{33}$ of $60 \mathrm{pC} / \mathrm{N}$, dielectric constant of 865 and dielectric loss of $5.45 \%$. The peak ME coefficient of $195 \mathrm{mV} / \mathrm{cm}$ Oe was observed at 454 Oe. This magnitude is much higher than that obtained for random polycrystalline sintered composites ${ }^{15}$ and about 130 times higher than that reported for the $\mathrm{BaTiO}_{3}-\mathrm{CoFe}_{2} \mathrm{O}_{4}$ coreshell composites. ${ }^{10}$ The increase in the ME coefficient $(\alpha)$ could be interpreted using the relationship proposed by Zubkov $^{16}$ as

$$
\begin{aligned}
\alpha_{E} & =(d E / d H)_{\mathrm{comp}}=(d S / d H)_{\mathrm{comp}}(d E / d S)_{\mathrm{comp}} \\
& =m_{v}(d S / d H)_{\text {ferrite }}\left(1-m_{v}\right)(d E / d S)_{\text {piezoelectric }}
\end{aligned}
$$

where $m_{v}$ is the volume fraction of ferrite, $(d E / d S)$ is the inverse of change in strain per unit electric field, and $(d S / d H)$ is the change in strain per unit magnetic field. Equation (1) shows that ME coefficient can be maximized by transferring large fraction of the strain generated in the ferrite on to the piezoelectric. The core-shell composite with good interface bonding and minimum misfit strain provides effective microstructure for this to occur. Assuming the deformation is occurring along the thickness direction and electric field is measured along the same direction, Eq. (1) can be approximated as

$$
\alpha_{E}=m_{v}(d S / d H)_{\text {ferrite }}\left(1-m_{v}\right)\left(g_{33} C_{33}\right)_{\text {piezoelectric }},
$$

where $g$ is piezoelectric voltage constant and $C$ is the stiffness. The magnitude of $(d S / d H)$ for common ferrite materials is of the order of $1 \times 10^{-9} \mathrm{~m} / \mathrm{A}\left(=79.577 \times 10^{-9} \mathrm{Oe}^{-1}\right)$. Thus, if the stiffness and voltage constant for composite are known then ME coefficient can be determined.

Further, it was found that in the dc bias range of 265 to $617 \mathrm{Oe}$, the ME coefficient remained above $175 \mathrm{mV} / \mathrm{cm} \mathrm{Oe}$, which is almost $90 \%$ of the peak value. This data is quite different as compared to that obtained for bulk particulates which exhibit sharp peaks. This wide bandwidth offers significant advantages for sensing applications. This may be due to the hard nature (larger coercive field) of the magnetic phase. The coercive field was of the order of 216 Oe for the core-shell structure. Above this field, the magnetization rotation contributes toward magnetostrictive strain. According to the theory developed from the cause-and-effect relation be- tween magnetization and magnetostriction hysteresis, remanence ratio $R\left(=M_{r} / M_{s}\right)$ is given as: $R=\left(H_{c} M_{s} / n C \lambda_{s}^{2}\right)^{1 /(2 n-1)}$ for $0 \leq R \geq 1$, where $H_{c}$ is the coercive field, $M_{s}$ is the saturation magnetization, $C$ is Young's modulus, $\lambda_{s}$ is the isotropic or average magnetostriction constant, and $n$ is of the order of $2{ }^{17,18}$ This relationship shows that higher magnetostriction can be maintained by maximizing the $H_{c} M_{s}$ coefficient as observed in Fig. 2(d). Above 617 Oe the ME coefficient drops as the piezomagnetic coefficient starts to drop rapidly.

\section{SUMMARY}

In summary, this letter reports the ME measurements on the core-shell sintered composites in the system PZT-NF. The results show that this structure can provide a large magnitude of ME coupling over a wide bandwidth.

\section{ACKNOWLEDGMENTS}

The authors gratefully acknowledge the financial support from (U.S.) Army Research Office.

${ }^{1}$ J. Van Suchetelene, Philips Res. Rep. 27, 28 (1972).

${ }^{2}$ G. R. Harshe, "Magnetoelectric effect in piezoelectric: Magnetostrictive composite," Ph.D. thesis, Pennsylvania State University, 1991.

${ }^{3}$ J. Ryu, S. Priya, and K. Uchino, J. Electroceram. 8, 107 (2002).

${ }^{4}$ S. Dong, J. Li, and D. Viehland, Appl. Phys. Lett. 83, 2265 (2003).

${ }^{5}$ S. Dong, J. Zhai, J. Li, and D. Viehland, Appl. Phys. Lett. 89, 252904 (2006).

${ }^{6}$ G. Srinivasan and Y. K. Fetisov, Ferroelectrics 342, 65 (2006).

${ }^{7}$ R. Zhang, M. Wang, N. Zhang, and G. Srinivasan, Acta Phys. Sin. 55, 2548 (2006).

${ }^{8}$ Z. Shi, J. Ma, Y. H. Lin, and C. W. Nan, J. Appl. Phys. 101, 043902 (2007).

${ }^{9}$ S. Dong, J. Zhai, J. Li, D. Viehland, and M. I. Bichurin, Appl. Phys. Lett. 89, 243512 (2006)

${ }^{10}$ V. Corral-Flores, D. B. Baques, D. C. Flores, and J. A. M. Aquino, J. Appl. Phys. 99, 08J503 (2006).

${ }^{11}$ Y. S. Koo, T. Bonaedy, K. D. Sung, J. H. Jung, J. B. Yoon, Y. H. Jo, M. H. Jung, H. J. Lee, T. Y. Koo, and Y. H. Jeong, Appl. Phys. Lett. 91, 212903 (2007).

${ }^{12}$ R. A. Islam, J. Mater. Sci. 43, 3560 (2008)

${ }^{13}$ G. Rowlands, J. Phys. D 9, 1267 (1976).

${ }^{14}$ K. Uestuener, M. Katter, and W. Rodewald, IEEE Trans. Magn. 42, 2897 (2006).

${ }^{15}$ S. Priya, R. Islam, S. Dong, and D. Viehland, J. Electroceram. 19, 147 (2007).

${ }^{16}$ A. S. Zubkov, Elektrichestvo 10, 77 (1978).

${ }^{17}$ G. F. Dionne and R. G. West, Appl. Phys. Lett. 48, 1488 (1986).

${ }^{18}$ G. F. Dionne and R. G. West, J. Appl. Phys. 61, 3868 (1987). 